\title{
Kinetic Modelling and Scaled-up Experimental Studies of Microalgal Fuels and Chemicals Production
}

DOI:

10.1016/B978-0-444-63965-3.50474-8

\section{Document Version}

Accepted author manuscript

Link to publication record in Manchester Research Explorer

\section{Citation for published version (APA):}

Bekirogullari, M., Pittman, J., \& Theodoropoulos, C. (2017). Kinetic Modelling and Scaled-up Experimental Studies of Microalgal Fuels and Chemicals Production. In European Symposium on Computer-Aided Process Engineering https://doi.org/10.1016/B978-0-444-63965-3.50474-8

\section{Published in:}

European Symposium on Computer-Aided Process Engineering

\section{Citing this paper}

Please note that where the full-text provided on Manchester Research Explorer is the Author Accepted Manuscript or Proof version this may differ from the final Published version. If citing, it is advised that you check and use the publisher's definitive version.

\section{General rights}

Copyright and moral rights for the publications made accessible in the Research Explorer are retained by the authors and/or other copyright owners and it is a condition of accessing publications that users recognise and abide by the legal requirements associated with these rights.

\section{Takedown policy}

If you believe that this document breaches copyright please refer to the University of Manchester's Takedown Procedures [http://man.ac.uk/04Y6Bo] or contact uml.scholarlycommunications@manchester.ac.uk providing relevant details, so we can investigate your claim.

\section{OPEN ACCESS}


Antonio Espuña, Moisès Graells and Luis Puigjaner (Editors), Proceedings of the $27^{\text {th }}$ European Symposium on Computer Aided Process Engineering - ESCAPE 27

October $1^{\text {st }}-5^{\text {th }}, 2017$, Barcelona, Spain (C) 2017 Elsevier B.V. All rights reserved.

\title{
Kinetic Modelling and Scaled-up Experimental Studies of Microalgal Fuels and Chemicals Production
}

\author{
Mesut Bekirogullari ${ }^{\mathrm{a}}$, Jon K. Pittman ${ }^{\mathrm{b}}$ and Constantinos Theodoropoulos ${ }^{\mathrm{a},{ }^{*}}$ \\ ${ }^{a}$ School of Chemical Engineering and Analytical Science, Biochemical and Bioprocess \\ Engineering Group, University of Manchester, M13 9PL, UK \\ ${ }^{b}$ School of Earth and Environmental Sciences, University of Manchester, M13 9PL, UK \\ *k.theodoropoulos@manchester.ac.uk
}

\begin{abstract}
Microalgal lipid production is a promising long-term feedstock for the biofuels industry. In this study, photoautotrophic microalgal growth in open ponds has been investigated for the purpose of developing a kinetic model for the optimization and scale-up of the microalgae cultivation process. A comprehensive kinetic modelling approach for Chlamydomonas Reinhardtii growth in open pond has been developed to predict biomass growth and lipid accumulation under simultaneous influence of nitrogen, temperature and light intensity. The kinetic model is based on a multiplicative model approach that assumes equal contribution of the aforementioned limiting factors. The developed model has been used in conjunction with in-house obtained experimental data to estimate model parameters and ultimately to enable the optimization of the microalgae cultivation in raceway open pond batch systems. Experiments were performed in $2 \mathrm{~m}^{3}$ open raceway ponds to define kinetic parameters of the scale-up level process manifested by nutrient and environmental parameters such as nitrogen, light and temperature. Scaled-up process simulations using such a comprehensive modelling framework will improve the competitiveness and sustainability of the microalgal biodiesel industry and will also enable the system industrial applicability and commercialization.
\end{abstract}

Keywords: microalgae, bench scale, raceway pond, multi-parameter quantification.

\section{Introduction}

Microalgae are photosynthetic microorganisms that carry out the same mechanism as higher plants in converting light and $\mathrm{CO}_{2}$ into value added products such as lipids carbohydrates, which can be directly used as high-value bioactives, or be utilized to produce biodiesel (Han et al., 2015). Due to depletion of known fossil fuels sources and environmental concerns, the worldwide interest in alternative fuels has increased (Raupach et al., 2007). Among all renewable feedstocks use of microalgae as feedstock for the production of biofuels has gained vast interest due to its outstanding characteristics such as high lipid content, rapid growth rate and non-competition with agricultural land and simple growing needs (light, sugars $\mathrm{N}, \mathrm{P}, \mathrm{K}$ and $\mathrm{CO}_{2}$ ) compared to other terrestrial plants (Driver, 2014). However, microalgal biodiesel production is currently too expensive to compete with the conventional plant sources and fossil diesel production (Chisti, 2007). In order to improve the competitiveness and sustainability of microalgal biodiesel industry, it is necessary to improve biomass productivity and lipid 
productivity by defining optimal growth conditions (Bekirogullari et al., 2017). Utilization of kinetic models is one of the useful optimization approaches which can be used for the optimization and scaled-up of the microalgae cultivation process. Microalgal growth is simultaneously influenced by various nutrients and environmental factors such as carbon source, nitrogen, light intensity, temperature and $\mathrm{pH}$. In order for a mathematical model to be able to predict the biomass growth and lipid accumulation and also to enable optimization process to be carried out, it is essential to account for the simultaneous effect of these parameters.

In this work we develop a detailed kinetic model that accounts for the simultaneous influence of nitrogen, light intensity, temperature and $\mathrm{pH}$ to be used in conjunction with in-house obtained experimental data to enable the optimization and the control of the microalgae growth in raceway open pond batch systems. Experiments were conducted using the well-studied chlorophyte microalgal species Chlamydomonas reinhardtii in $2 \mathrm{~m}^{3}$ open raceway ponds to efficiently define kinetic parameters that are crucial for accurate process simulations and validation of the developed model for the scale-up level. Moreover, an in-house developed stochastic algorithm, based on Simulated Annealing (SA), is linked to a deterministic MATLAB optimization function (fmincon) for the estimation of key parameters used in the large-scale raceway open pond (Bekirogullari et al., 2016). To the best of our knowledge, this work is the first to attempt to develop a kinetic model in conjunction with experimental data to predict the complex behaviour of large-scale raceway open pond. Such an integrated experimentalcomputational framework can be exploited for the robust system design and also to provide insights on biomass growth and lipid production metabolism.

\section{Materials and Methods}

\subsection{Strain and Culture Conditions}

Chlamydomonas Reinhardtii (CCAP 11/32C) was obtained from the Culture Collection of Algae and Protozoa, UK and cultivated in $2 \mathrm{~m}^{3}$ raceway open pond. The strain was originally adapted to grow in Tris-acetate-phosphate (TAP) medium. In order for the strain to grow in a fertilizer medium, which consisted of diluted Wilko liquid tomato feed $\left(100 \mathrm{mgl}^{-1} \mathrm{NH} 4-\mathrm{N}, 55 \mathrm{mgl}-1 \mathrm{PO}_{4}^{-3}-\mathrm{P}\right)$, an adaption process carried out prior to experiments in open pond. The adaption process was based on increasing the fertilizer concentration and decreasing the TAP concentration in the TAP growth media gradually. Preculture of the strain was carried out in an environmentally-controlled growth chamber at $25^{\circ} \mathrm{C}$ and $125 \mu \mathrm{Em}^{-2} \mathrm{~s}^{-1}$ light intensity using $250 \mathrm{~mL}$ conical flasks containing $150 \mathrm{~mL}$ of TAP medium on an orbital shaker at $120 \mathrm{rpm}$ for 45 days. The preculture of the strain was stopped when the strain was adapted to grow in fertilizer medium and sufficient cell concentration was achieved. An algal inoculum of $1 \mathrm{~L}$ was then added to raceway open ponds $\left(2 \mathrm{~m}^{3}\right)$ which contains $500 \mathrm{~L}$ of culture medium. The growth process continued for 45 days and daily samples were taken to analyse the biomass growth rate, lipid accumulation, $\mathrm{pH}$ change and nutrient consumption.

The samples were centrifuged for $3 \mathrm{~min}$ at $3000 \mathrm{~g}$ in an Eppendorf Centrifuge 5424 to define biomass concentration in terms of dry cell weight (DCW). The obtained wet pellet dried overnight at $70{ }^{\circ} \mathrm{C}$ and weighed to determine $\mathrm{DCW}$. The $\mathrm{pH}$ of the samples was examined through the use of a bench type $\mathrm{pH}$ meter (Denver UltraBasic Benchtop Meters, USA). Daily light intensity was measured by use of a light meter (Fisher Scientific Light Meter, Leicestershire, UK). The supernatant and dry biomass of the 
Kinetic Modelling and Scaled-up Experimental Studies of Microalgal Fuels and Chemicals Production

samples were stored at $-20^{\circ} \mathrm{C}$ for the quantification of lipid accumulation and nitrogen consumption.

The total dissolved nitrogen concentration in the growth media was quantified by the use of Total Organic Carbon / Total Nitrogen analyzer (TOC/TN). The lipid concentration in the dried algal biomass was quantified by extracting the lipid through use of Soxhlet Solvent Extraction by Using Soxtec. The freeze-dried algal biomass was homogenized through a double cycle of liquid $\mathrm{N}_{2}$ immersion and pulverization in a mortar with pestle. After placing the pulverized biomass into cellulose extraction thimbles and locating into Soxtec unit, the following procedure was then followed: boiling for 2 hours, rinsing for $40 \mathrm{~min}$ and solvent recovery $20 \mathrm{~min}$. Hexane (ACS spectrophotometric grade, $\geq 98.5 \%$, Sigma Aldrich, Dorset, UK) was chosen as solvent and the extraction temperature was set to $155^{\circ} \mathrm{C}$. The extracted lipid with the use of Soxtec 1043 were dried at $100^{\circ} \mathrm{C}$ for $1 \mathrm{~h}$, placed in vacuum applied desiccator for $1 \mathrm{~h}$, and finally weighed to define lipid concentration gravimetrically.

\section{Kinetic Modelling}

A comprehensive kinetic model was constructed considering simultaneous simulation of carbon substrate, nitrogen, light intensity and $\mathrm{pH}$ in bench scale microalgal growth (Bekirogullari et al., 2017). The model was validated and applied to determine optimal growth conditions, which lead to maximized biomass productivity and oil productivity (Bekirogullari et al., 2017). The model was then adapted to be used to predict the complex nature of large-scale raceway open pond systems. Based on that study, here we have developed an extended ordinary differential equation- (ODE) based model to describe the microalgal growth and oil production processes.

As it is known the nitrogen depletion favors the oil accumulation while reducing the biomass growth (Bekirogullari et al., 2017). Due to the contrasting effect of nitrogen, two different expressions were employed to describe the oil-free biomass growth and lipid accumulation. Specific oil-free biomass growth, $\mu_{X}$, and lipid accumulation, $\mu_{L}$, are expressed by a multiplicative (triple) substrate expression:

$$
\begin{gathered}
\mu_{X}=\mu_{X \max } \cdot \frac{N}{N+K_{X N}+\frac{N^{2}}{K_{i X N}}} \cdot \frac{I}{I+K_{X I}+\frac{I^{2}}{K_{i X I}}} \cdot f(T) \\
\mu_{L}=q_{L \max } \cdot \frac{K_{i L N}}{N+K_{i L N}} \cdot \frac{I}{I+K_{L I}+\frac{I^{2}}{K_{i L I}}} \cdot f(T)
\end{gathered}
$$

where $\mu_{X \max }$ is the maximum specific growth rate of oil-free biomass production $\left(g L^{-1}\right), q_{L \max }$ is the maximum specific rate of lipid production $\left(g L^{-1}\right), N$ is the nitrogen concentration $\left(g L^{-1}\right), K_{X N}$ is the saturation constants $\left(g L^{-1}\right), K_{i X N}$ and $K_{i L N}$ are the inhibition constants of nitrogen $\left(g L^{-1}\right), I$ is the local light intensity $\left(\mu \mathrm{Em}^{-2} \mathrm{~s}^{-1}\right), K_{L I}$ and $K_{X I}$ are the saturation constants $\left(g L^{-1}\right)$, and $K_{i X I}$ and $K_{i L I}$ are the inhibition constants of light intensity.

The light intensity distribution, $I$, is given Beer-Lambert law:

$$
f(I)=I_{0} \exp (-\sigma \mathrm{Xl})
$$


where $l$ is the distance between the local light position and the external surface of the growth system (the value of $l$ is given in Table 1), $I_{0}$ is the local light intensity $\left(\mu \mathrm{Em}^{-2} \mathrm{~s}^{-1}\right)$ and $\sigma$ is the molar extinction coefficient.

Eq. (3) holds for continues light illumination. In order to account for variations in the photoperiod ( 8 hours of light and 16 hours of dark), we have obtained the following quadratic equation for the photoperiodic light change:

$$
I_{0}=-1101 * C^{2}+733.5 * C
$$

where $C$ is the proportion of light/dark cycle $(0 \leq C \leq 1)$.

The values of the quadratic equation's variables were defined based on the measured data. The light intensity was measured for 45 days and the mean value was found to be $122 \mu \mathrm{Em}^{-2} \mathrm{~s}^{-1}$.

The effect of temperature is expressed as a modified Arrhenius equation in order to account for both saturation and inhibition (Bitaubé Pérez et al., 2008):

$$
f(T)=A_{0} \exp \left[\frac{-E_{a}}{R}\left(\frac{1}{T}-\frac{1}{T_{0}}\right)\right]-B_{0} \exp \left[\frac{-E_{b}}{R}\left(\frac{1}{T}-\frac{1}{T_{0}}\right)\right]
$$

where the first part of the equation on the right hand side expresses the promotion effect while second part shows the inhibition effect of temperature. $E_{a}$ and $E_{b}$ are the activation energies of growth and cellular degradation, respectively $\left(\mathrm{kcal} \mathrm{mol}^{-1}\right), R$ is the gas constant $\left(\mathrm{kcal} \mathrm{mol}^{-1}\right), T$ is the incubation temperature $(K), T_{0}$ is the reference temperature $(K)$, and $A_{0}$ and $B_{0}$ are the corresponding frequency factors $\left(h^{-1}\right)$.

Eq. (5) can only be applied to constant incubation temperature. To account for the periodic change of temperature $\left( \pm 4^{\circ} \mathrm{C}\right)$, we made the following quadratic formula:

$$
T_{0}=-36.036 * C^{2}+24 * C+285
$$

where $C$ is the proportion of day/night period $(0 \leq C \leq 1)$.

The method used in Eq. (4) to define values of the variables was also employed here to determine the values of the Eq. (6). The daily temperature was measured for 45 days and the average value was found to be $16^{\circ} \mathrm{C}$.

The kinetic model developed in this work consists of a set of ODEs used to simulate microalgal biomass growth, lipid accumulation, nitrogen consumption and $\mathrm{pH}$ change. The oil-free biomass growth rate is described as:

$$
\frac{d X}{d t}=\mu_{X} \cdot X
$$

The oil accumulation rate is expressed as:

$$
\frac{d L}{d t}=\mu_{L} \cdot X
$$

The nitrogen consumption rate is given by: 
Kinetic Modelling and Scaled-up Experimental Studies of Microalgal Fuels and Chemicals Production

$$
\frac{d N}{d t}=-\frac{1}{Y_{X / N}} \cdot \frac{d X}{d t}
$$

The $\mathrm{pH}$ change rate of microalgal systems is proportional to the nitrogen consumption rate and it is given by:

$$
\frac{d H}{d t}=-k_{H} \cdot \frac{d N}{d t}
$$

Consequently, the developed model consists of 4 ODEs (Eq. (7) - Eq.(10)), corresponding to 4 state variables describing the oil-free biomass growth and lipid accumulation as well as nitrogen consumption and $\mathrm{pH}$ change of the systems.

\section{Results and Discussion}

An in-house developed stochastic optimization algorithm (SA) is coupled with a deterministic MATLAB optimization function (fmincon) for the estimation of key parameters used in the large-scale raceway open pond. The developed model presented in section 3 was fitted to a duplicate set of large-scale open pond experiments, performed on fertilizer media containing $100 \mathrm{mgl}^{-1}$ NH4-N (Figure 1). The obtained kinetic parameter values are tabulated in Table 1.

As we can see in Figure 1, the model was capable of predicting the experimentally obtained time profiles of biomass growth, lipid production and nitrogen consumption with high accuracy precision. The developed model and the obtained kinetic parameters are yet to be validated. The validation of the model will be carried out against a different a set of conditions where nitrogen concentration will be different and environmental factors will change based on daily weather conditions. After carrying out the validation study, we believe that this detailed multiplicative kinetic model proposed here can be utilized for accurate predictions of the dynamic behaviour of large-scale raceway open pond experiments and it can also be used in optimization studies to obtain optimal growth parameters leading to maximum biomass growth and lipid accumulation.
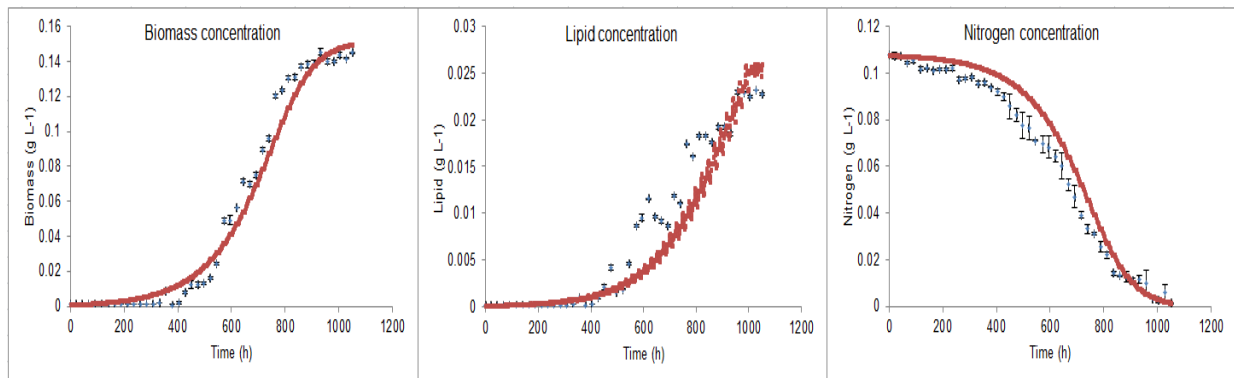

Figure 1 Fitting of model predictions (lines) to experimental data (symbols with error bars) for: (a) biomass, (b) lipid concentration, (c) $\mathrm{N}$ consumption using $100 \mathrm{mgl}^{-1} \mathrm{NH} 4-\mathrm{N}$. 
Table 1 Model kinetic parameters

\begin{tabular}{clclcl}
\hline Parameter & Value & Parameter & Value & Parameter & Value \\
\hline$\mu_{X \max }$ & 0.1767 & $E_{b X}$ & 17.8247 & $E_{b X}$ & 35.8541 \\
$K_{X N}$ & 0.1524 & $q_{L \max }$ & 0.22 & $\sigma$ & 14.2469 \\
$K_{i X N}$ & 0.3281 & $K_{i L N}$ & 79.8049 & $Y_{X} / N$ & 1.4040 \\
$K_{X I}$ & 5.5159 & $K_{L I}$ & 0.9268 & $k_{H}$ & 5.1330 \\
$K_{i X I}$ & 141.5918 & $K_{i L I}$ & 113.7640 & $l$ & 0.25 \\
$A_{0 X}$ & 0.5241 & $A_{0 X}$ & 0.1248 & $R$ & 0.00198588 \\
$E_{a X}$ & 3.2503 & $E_{a X}$ & 3.2137 & & \\
$B_{0 X}$ & 0.3766 & $B_{0 X}$ & 0.3340 & & \\
\hline
\end{tabular}

\section{Conclusions}

Few studies have attempted to model microalgal biomass growth and lipid accumulation in open ponds but none of these studies have modelled the biomass growth and lipid accumulation as two different state variables to take advantage of the promotion effect of nitrogen towards lipid accumulation. Here, we developed a multi-parameter kinetic model to describe biomass growth and lipid production as well as nitrogen consumption and $\mathrm{pH}$ change. The formulated model considers simultaneous and antagonistic effects of three growth limiting factors, nitrogen, light intensity and temperature on the rate of biomass growth and lipid accumulation. An experimental study was undertaken in $2 \mathrm{~m}^{3}$ raceway open ponds and it was used in conjunction with the developed model to estimate the kinetic parameters that are essential for precise system simulations. Such detailed kinetic models can be exploited for the robust design and control of microalgal lipid production technologies, which can bring microalgal biomass production technology one step closer to commercialization and industrial applicability.

\section{References}

Bekirogullari, M., Fragkopoulos, I. S., Pittman, J. K. \& Theodoropoulos, C. 2017. Production of lipid-based fuels and chemicals from microalgae: An integrated experimental and model-based optimization study. Algal Research, 23, 78-87.

Bekirogullari, M., Pittman, J. \& Theodoropoulos, C. 2016. Experimental Studies and Model Based Optimisation of Microalgal Production of Fuels and Chemicals. In: Zdravko, K. \& Miloš, B. (eds.) Computer Aided Chemical Engineering. Elsevier.

Bitaubé Pérez, E., Caro Pina, I. \& Pérez Rodríguez, L. 2008. Kinetic model for growth of Phaeodactylum tricornutum in intensive culture photobioreactor. Biochemical Engineering Journal, 40, 520-525.

Chisti, Y. 2007. Biodiesel from microalgae. Biotechnol Adv, 25.

Driver, T., Bajhaiya, A. \& Pittman, J. K. 2014. Potential of Bioenergy Production from Microalgae. Current Sustainable/Renewable Energy Reports, 1, 94-103.

Han, F., Pei, H., Hu, W., Song, M., Ma, G. \& Pei, R. 2015. Optimization and lipid production enhancement of microalgae culture by efficiently changing the conditions along with the growth-state. Energy Conversion and Management, 90, 315-322.

M. R. Raupach, G. Marland, P. Ciais, C. Le Quéré, J. G. Canadell, G. Klepper \& C. B. Field, 2007; Proceedings of the National Academy of Sciences, 104, 10288-10293. 